\title{
Modulation of Muscle Atrophy, Fatigue and MLC Phosphorylation by MuRF1 as Indicated by Hindlimb Suspension Studies on MuRF1-KO Mice
}

\author{
Siegfried Labeit, ${ }^{1}$ Christine H. Kohl, ${ }^{1}$ Christian C. Witt, ${ }^{1}$ Dittmar Labeit, ${ }^{1}$ Jeong Jung, ${ }^{2}$ \\ and Henk Granzier ${ }^{2}$ \\ ${ }^{1}$ Department of Integrative Pathophysiology, Universitätsmedizin Mannheim, University of Heidelberg, Theodor-Kutzer-Ufer 1-3, \\ 68167 Mannheim, Germany \\ ${ }^{2}$ Department of Physiology, University of Arizona, Tucson, AZ 85724, USA
}

Correspondence should be addressed to Siegfried Labeit, labeit@embl.de

Received 20 February 2010; Accepted 13 April 2010

Academic Editor: Aikaterini Kontrogianni-Konstantopoulos

Copyright (C) 2010 Siegfried Labeit et al. This is an open access article distributed under the Creative Commons Attribution License, which permits unrestricted use, distribution, and reproduction in any medium, provided the original work is properly cited.

\begin{abstract}
MuRF1 is a member of the TRIM/RBCC superfamily, a gene family that encompasses a large variety of proteins, all sharing the conserved TRIM (Tripartite Motive) sequential array of RING, B-box, and coiled-coil domains. Within this family, MuRF1(also named TRIM63) is a specialized member that contributes to the development of muscle atrophy and sarcopenia. Here we studied MuRF1's role in muscle atrophy during muscle unloading induced by hindlimb suspension. Consistent with previous studies, we found that MuRF1 inactivation leads to an attenuated muscle atrophy response. The amount of protection was higher as compared to the denervation model, and within the 10 day-suspension period the soleus muscle was spared from atrophy in MuRF1-KO mice. Contractility studies on hindlimb suspended muscle tissues suggested that MuRF1's functions extend beyond muscle trophicity and implicate MuRF1 in muscle fatigue and MLC phosphorylation control: soleus muscle from MuRF1-KO mice fatigued significantly faster and in addition showed a reduced posttetanic twitch potentiation. Thus the present work further established the role of MuRF1 in muscle atrophy and for the first time shows that MuRF1 plays a role in muscle fatigue and twitch potentiation.
\end{abstract}

\section{Introduction}

Skeletal muscle tissues can be extensively remodeled, both structurally and functionally (for reviews see $[1,2]$ ). Such remodeling takes places, for example, in response to exercise or passive muscle stretch and results in large changes in metabolic activity, fiber-type, and force generation (for reviews see $[3,4])$. Increased force production is due to an increase in fiber diameter, an adaptation referred to as hypertrophy. In contrast, when muscle is unloaded, as occurs, for example, in space flight or in bedridden patients, fiber diameter is rapidly reduced, a state referred to as atrophy. Muscle atrophy also accompanies the natural process of aging, and is then referred to as sarcopenia, and leads to a progressive and large reduction in muscle strength as ageing progresses [5]. Skeletal muscle atrophy is also a debilitating response to starvation and many systemic diseases including diabetes, cancer, and renal failure [6]. Thus understanding the mechanisms that underlie atrophy is clinically highly significant for space flight, patients that have a wide range of diseases or that are bedridden, and to understand and perhaps ameliorate sarcopenia.

Because of the clinical and health-economic importance of sarcopenia, the molecular pathways involved have attracted wide interest. Comparative studies on healthy and wasting muscle tissues identified numerous mechanisms involved in the regulation of muscle trophicity, including 
the calcineurin-NFAT pathway (stimulated by the calcium influx into the sarcoplasm), the mTOR/p70S6K pathway (modulated by myofibrillar stretch), and stress signaling (modulated by p38/ERK/MEK kinase pathways, see for example $[7,8]$ ). More recently, molecular insights into the mechanisms counteracting these trophic pathways could be obtained. Importantly, atrogin-1 and MuRF1 genes were found to be transcriptionally upregulated during diverse states of muscle wasting $[9,10]$. Furthermore, studies using atrogin-1 and MuRF1-deficient mouse models further supported a potential causal role of these two genes in the development of muscle atrophy: Knock-out mouse models of atrogin-1 and MuRF1 develop about 50\% less muscle atrophy in quadriceps muscle after lesion of its innervation [11]. Mechanistically, it was proposed that these two genes act as atrogins via the control of proteasome-dependent degradation of muscle proteins, a concept, that is supported in vitro by the enzymatic E3 ligase activity of expressed MuRF1 protein. Therefore, an upregulation of these E3 ubiquitin ligase activities during muscle wasting would enhance the UPS-dependent degradation of muscle proteins (for review see [12]).

Current studies on MuRF1 and atrogin-1 are therefore focused on the identification of their in vivo targets. In vitro studies demonstrated that MuRF1 efficiently catalyzes the addition of multiubiquitin groups to troponin-I (TnI), myosin, and actin $[9,10]$. However, target recognition in vivo is likely to be more selective and to be regulated by yet unknown factors. For example, a recent study using a CreLox-recombinase induced conditional $\mathrm{KO}$ model where MuRF1 activity can be downregulated in adult mice suggested that myosin, actin, and TnI are not preferred in vivo targets of MuRF1 [13]. Rather, a partial protection of MLC2 and myosin binding protein $\mathrm{C}$ turnover after denervation was observed in an MuRF1-deficient conditional background [13]. Consistent with the idea that MuRF1 target recognition in vivo is still poorly understood is the finding that overexpression of MuRF1 in skeletal muscles alone is not sufficient to cause muscle atrophy [14]. Instead of muscle wasting, overexpression of MuRF1 resulted in a pertubation of muscle metabolism and insulin signaling [14]. This raises the possibility that MuRF1 could also play a role in muscle trophicity via more complex pathways that include metabolic pathways, rather than the direct degradation of myofibrillar proteins.

Here were we used hindlimb suspension as an alternative model to study MuRF1's role in muscle atrophy (in this model muscle maintains its innervation), and studied fiber type distribution, muscle force, and fatigue resistance during repetitive stimulation. Results indicate that muscles from suspended wildtype (wt) and MuRF1-KO (KO) mice have comparable contractile characteristics, suggesting that degradation of myofibrillar proteins by MuRF1 is unlikely. However, inactivation of MuRF1 resulted after hindlimb suspension in a reduced fatigue tolerance and reduced posttetanic potentiation, linking MuRF1 to MLC phosphorylation. These findings implicate that MuRF1's functional roles in muscle extend beyond a pathophysiological role in causing muscle atrophy.

\section{Methods}

2.1. Animals and Hindlimb Suspension. All mice were bred in-house and genotyped by standard methods. MuRF1 null mice were on a C57/BL6 background and MuRF1 gene disruption by homologous recombination was as previously described [15]. Animals were housed in standard cages in an animal room with controlled environmental conditions and maintained on standard food and water ad libitum. To suspend the hindlimbs, the tail of each mouse was placed in a harness, which was used to elevate the pelvis so that the feet of the hindlimbs did not contact the cage floor. The harness consisted of SkinTrac (a commercially available adhesive tape) in which the entire length of the tail was trapped. The distal end of the SkinTrac was attached to a paperclip which was then attached to a swivel on a plexiglass cross-bar. The cross-bar was positioned $\sim 15 \mathrm{~cm}$ above the cage floor. In this way, the hindlimb-suspended mice were able to freely move around the cage on their front limbs and had unlimited access to food and water. The hindlimb suspension lasted 10 days, afterwards one group of mice was sacrificed whereas another group was allowed to recover for 5 days (reload phase).

2.2. Characterization of Dissected Soleus Muscle. After 10 days of hindlimb suspension mice were anaesthetized with isoflurane and sacrificed via cervical dislocation. The soleus muscle was then rapidly dissected in an oxygenated bath on ice. After dissection, a suture was attached to the tendon at the proximal and distal ends of the muscle. The muscle was then attached, via sutures, to an Aurora 1200A Intact Muscle Test System and was placed in an oxygenated physiological solution at $30^{\circ} \mathrm{C}$. Next, the optimal twitch length $\left(L_{0}\right)$ was determined at $30^{\circ} \mathrm{C}$ by incrementally lengthening the muscle until gains in twitch force reached a plateau. After three minutes, the muscles were then passively stretched to four different lengths $\left(5 \%, 10 \%, 15 \%, 20 \%\right.$ of $\left.L_{0}\right)$ at a rate of $10 \% / \mathrm{sec}$, held at the respective length for 60 seconds and released then to $L_{0}$. Muscle strips were allowed to recover by waiting for 7 minutes between each stretch protocol. Seven minutes after the last stretch, a single twitch and tetanus was imposed on the muscle from $L_{0}$. A force-frequency protocol followed using the following frequencies in the following order $1,5,10,20,40,60,80,100$, and $150 \mathrm{~Hz}$, and waiting $30,30,60,90,120,120,120,120$ seconds, respectively, in between each activation. The muscle was then fatigued by stimulation at $60 \mathrm{~Hz}$ every three seconds for a total of 100 stimuli. The data from each experiment were analyzed with Aurora's DMA software, Microsoft Excel, and Kaleidagraph 3.6. Twitch and tetanus force and kinetics were determined using Aurora's DMA software. When the experiment was completed muscles were dried with blotting paper and were then weighed. The average cross-sectional area was obtained by dividing the weight by the specific gravity of muscle (1.06) and by the muscle length.

2.3. Immunohistochemistry. The primary antibodies used for immunostaining are commercially available: (1) monoclonal 
mouse anti-myosin heavy chain (MHC) II, clone MY-32 (1:1,000; cat\# M4276, Sigma) and (2) monoclonal mouse anti-skeletal myosin MHC I, clone NOQ7.5.4D (1:4,000; cat\# M8421, Sigma), and (3) MuRF1 was detected with specific antibodies as recently described [16]. The secondary antibodies used for immunostaining were (1) goat antimouse IgG-Cyanine Cy2 (1:100; cat\# 115-225-174, Jackson Lab), (2) donkey anti-chicken IgY-Cyanine Cy3 (1:1000; cat\# 703-165-155, Jackson Lab), (3) donkey anti-mouse Cyanine Cy3 (1:1000; cat\# 715-165-150, Jackson Lab), and (4) donkey anti-rabbit Cyanine Cy3 (1:100; cat\# 711165-152, Jackson Lab). Frozen cross-sections of muscles for immunostaining were fixed with $4 \%$ paraformaldehyde in $0.2 \mathrm{M}$ phosphate buffer $(\mathrm{PB})$ for 10 minutes at room temperature, washed with PBS 3 times for 3 minutes each and then blocked/permeabilized with $0.1 \mathrm{M}$ glycine/ $0.2 \%$ triton X-100 in phosphate-buffered saline (PBS) for 1 hour. Subsequently the slides were incubated with a solution containing the primary antibody, $3 \%$ normal goat serum and $0.3 \%$ triton $\mathrm{X}-100 / 0.1 \mathrm{M} \mathrm{PB}$ overnight in a moisture chamber $\left(4^{\circ} \mathrm{C}\right)$. After washing with $0.1 \mathrm{M}$ PBS (3 times for 10 minutes each), a solution containing the secondary antibody and $0.3 \%$ triton X-100/0.1 M PBS was added for 2 hours in a dark chamber. The slides were then washed in $0.1 \mathrm{M}$ PB (3 times for 10 minutes each) and mounted with Vectashield mounting medium for fluorescence with $4^{\prime}$, 6-diamidino-2-phenylindole (DAPI) (cat\# H-1200, Vector Labs) and coverslipped.

2.4. Quantitative and Morphometric Analysis. The quantitative and morphometric analysis were evaluated on a microscope (Nikon Eclipse E600, Fukuoka, Japan) equipped with a digital video camera and image software (Metamorph, Universal Imaging Corporation, Downingtown, USA) digitizing unit connected to a computer (Image Pro-plus, Media Cybernetic). For the determination of muscle fiber type I and II and cross-sectional area (CSA), a total of approximately 500 fibers per muscle per each group were studied. Three to four cross-sections of the soleus and muscles from different animals were analyzed in all groups. For classification of type I and type II fibers, positive immunolabeling for MHCI and MHCII antibodies were used, respectively. Fibers which were lightly labeled were considered intermediary.

2.5. Gel Electrophoresis and Western Blots. Protein extracts were prepared from frozen samples of soleus and tibialis cranialis muscles of 6 unsuspended, suspended and reloaded wild type mice. A total of $50 \mu \mathrm{g}$ of solubilized extracts were loaded on gel, and separated on $4-10 \%$ gradient SDS-acrylamide gels (Invitrogen) followed by transfer onto PVDF membranes. The protein levels of MuRF1 were determined with specific antibodies previously described $[17,18]$. After incubation with primary antibodies, specific bands were visualized by enzymatic chemiluminescence using horseradish-peroxidase-conjugated secondary antibodies, (Super Signal West Pico; Pierce, Bonn, Germany), that were quantified by densitometry using a onedimensional scan software package (Scanalytics, Rockville,
USA). Loading and blotting variability was monitored on duplicate Westernblots, reacted with antibodies specific for alpha-Tubulin (monoclonal rabbit anti-alpha-Tubulin antibody from Cellsignalling (\#2125); used at $1: 1,000)$.

2.6. Statistics. Multiple comparisons of mean values were performed with analysis of variance (ANOVA), for comparisons of only two groups, the unpaired $t$-test was used. For all comparisons, a $P<.05$ was considered significant.

\section{Results}

3.1. MuRF1 Induction during Hindlimb Suspension and Its Effect on Muscle Atrophy. First, we used the hindlimb suspension model to monitor MuRF1 protein expression by Western blots. For this, we compared unsuspended, suspended and reloaded muscles and included in our study soleus muscle (SO; because it has mixed-fiber types), as well the M. tibialis cranialis (TC, because it is an almost pure fastfiber type muscle). Our blots detected basal levels of MuRF1 expression also in all nonsuspended WT control samples (about 25\% of suspended protein levels; see Figure 1). Hindlimb suspension resulted in a severalfold upregulation of MuRF1 protein expression (3.9 fold in tibialis cranialis, 3.6-fold in soleus). This process was fully reversible: 5 days of reloading resulted in a return of MuRF1 expression to basal levels (Figure 1). Finally, our blot results were similar for tibialis and soleus muscle types (Figure 1).

Functionally, ten days of hindlimb suspension resulted in a $25 \%$ weight reduction of the soleus muscle. This effect was absent in the MuRF1-KO mouse (Table 1). We also determined the cross-sectional area of the muscles and found that the area was significantly reduced during hindlimb suspension of the wt mice $(P<.01)$ but that there was no change in the during hindlimb suspension of the KO mice $(P>.05)$ (Figure 2). We next measured the cross-sectional area of muscle fibers in the soleus muscle (measurements at $L_{0}$, see below), using immunohistochemistry to classify fibers as type I and II, because a recent study suggested that MuRF1 regulates type-II fiber trophicity in the denervation model [19]. We found that during hindlimb suspension of WT mice the cross-sectional area was significantly reduced in both type I and type II fibers (Figure 3, $P<.05$ ). A minor reduction of crosssectional area in MuRF1-KO animals was detectable but not significant. Fiber type distribution was not altered in the wt or in the MuRF1-KO soleus after hindlimb suspension. This conclusion is consistent with an SDS-PAGE based analysis of MHC isotypes in the respective samples (see Table 2). In sum, during hindlimb suspension the soleus muscle atrophies in WT mice, due to a reduction in the cross-sectional area of both type I and type II fibers. Soleus from MuRF1-KO mice seems to be affected only marginally.

3.2. Soleus Muscles from MuRF1-KO Mice Have Normal Contractility. Next, we studied contractile parameters of soleus muscle. Muscles were twitch activated and gradually stretched to $L_{0}$, the length at which twitch force is maximal. 


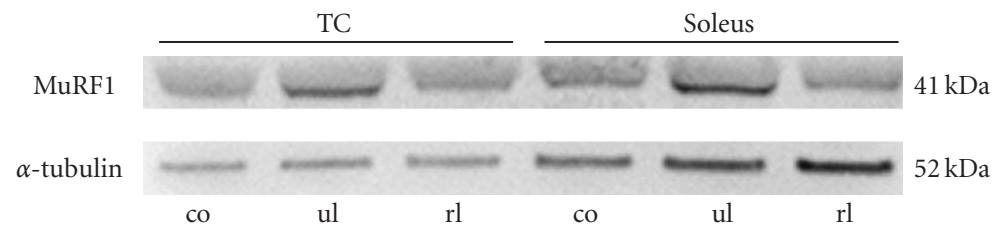

(a)

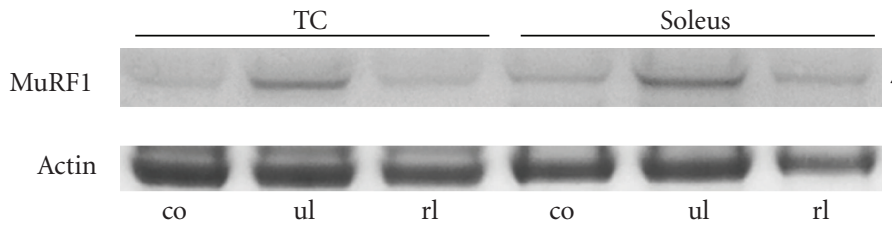

(b)

FIGURE 1: MuRF1 protein expression and hindlimb suspension. MuRF1 protein expression was monitored in tibialis cranialis (TC) and soleus (Sol.) muscles before (co; control), after unloading by hindlimb suspension (ul), and after reloading (rl). MuRF1 expression was compared relative to alpha-tubulin (a) or actin (b) as a control for loading and blotting. Low levels of MuRF1 protein were detected in control TC and Sol.; MuRF1 was severalfold elevated after unloading by hindlimb suspension in both muscle types and returned to nearly normal expression levels after 5 days of reloading $(\mathrm{rl})$.

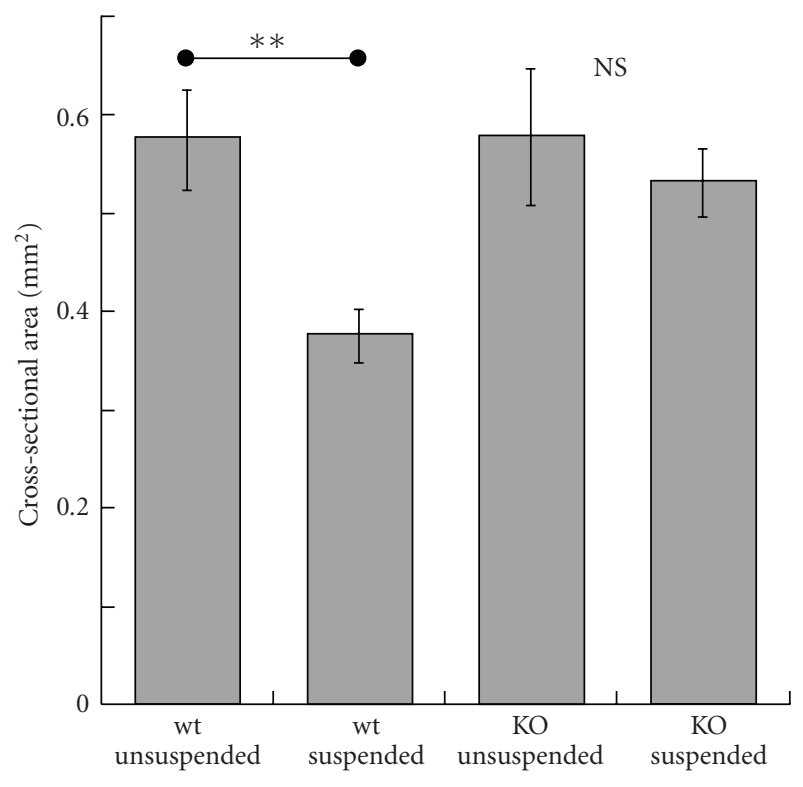

FIGURE 2: Cross-sectional analysis of soleus muscle. The crosssection of wt soleus muscle is significantly reduced during hindlimb suspension (wt sus), but not in the MuRF1-KO mice. “*” indicates statistical significance and "NS" its absence.

TABLE 1: Soleus weights (mg).

\begin{tabular}{lcc}
\hline & WT & KO \\
\hline Control & $6.9 \pm 0.5(8)$ & $6.2 \pm 0.6(6)$ \\
Susp. & $5.2 \pm 0.5(8)$ & $6.5 \pm 0.5(8)$ \\
$P$-value & 0.03 & NS \\
\hline
\end{tabular}

We then determined the force-frequency relation by imposing a 1 second pulse train at progressively higher frequency (see Section 2). No differences were found in the force frequency relation of the four studied groups (WT and $\mathrm{KO}$,
TABLE 2: Soleus fiber types based on MHC/SDS-PAGE typing.

\begin{tabular}{lccc}
\hline & & WT & KO \\
\hline \multirow{2}{*}{ Control } & Type I & $32.2 \pm 1.9(8)$ & $35.7 \pm 2.1(6)$ \\
& Type IIA+IIB & $67.8 \pm 3.9(8)$ & $64.3 \pm 4.1(8)$ \\
Susp. & Type I & $34.1 \pm 2.7(8)$ & $34.7 \pm 2.0(6)$ \\
$P$-value & Type IIA+IIB & $65.9 \pm 4.9(8)$ & $65.3 \pm 3.9(8)$ \\
\hline & & NS & NS
\end{tabular}

suspended and unsuspended). In all groups, muscle force reached a maximum at a stimulation frequency of $\sim 100 \mathrm{~Hz}$ and the half-maximal force was reached at a stimulation frequency of $\sim 35 \mathrm{~Hz}$. The identical force-frequency relations suggest that hindlimb suspension and the absence of MuRF1 does not affect calcium cycling.

We next studied the effect of hindlimb suspension on the maximal tetanic force (stimulation frequency $150 \mathrm{~Hz}$ ) in both wt and $\mathrm{KO}$ mice and found that this force was significantly reduced during hindlimb suspension of wt mice $(P<.01)$, with no effect in MuRF1-KO mice (see Figures $4(\mathrm{a})$ and 4(b)). However, when the maximal force was normalized to the cross-sectional area of the muscle, the obtained specific forces were not different amongst the 4 groups (see Figure 4(c)). Thus, the atrophy that occurs in wt mice fully explains the reduction in tetanic force during hindlimb suspension of wt mice. The absence of an effect of hindlimb suspension on both total force and specific force in MuRF1-KO mice (Figures 4(b) and 4(c)) is consistent with the absence of atrophy. Additionally, the finding that the force of MuRF1-KO mice is the same as that of unsuspended wt mice suggests that under our experimental conditions the absence of MuRF1 does not affect the functionality of the contractile proteins. This is also suggested by the absence of differences between wt and $\mathrm{KO}$ groups in the time required to reach maximal tetanic force and the force relaxation time when stimulation was terminated (results not shown). 
Control

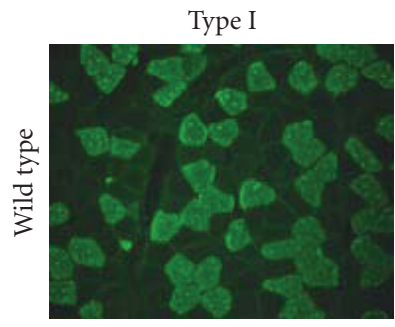

Type I

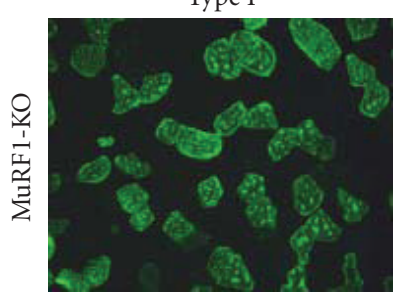

(a)

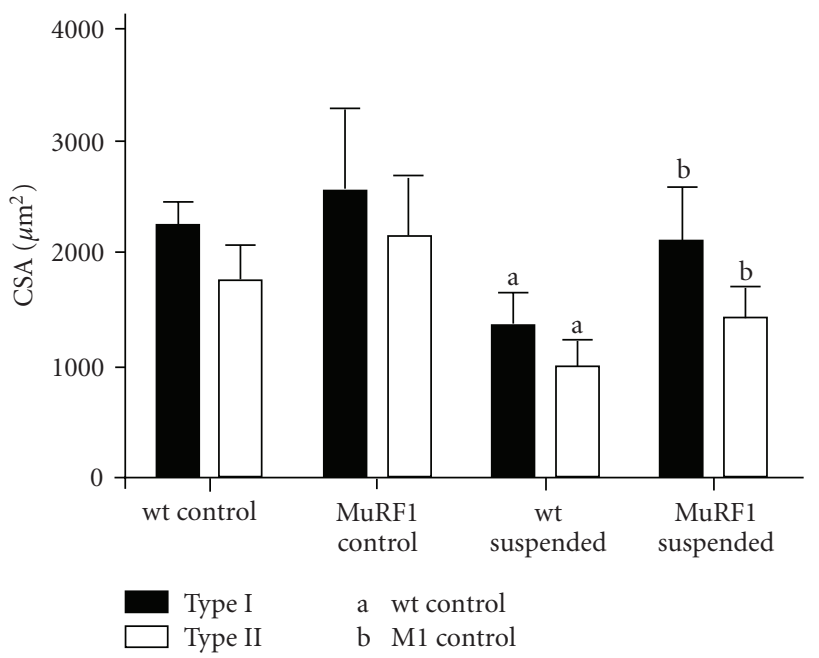

(c)

Suspended

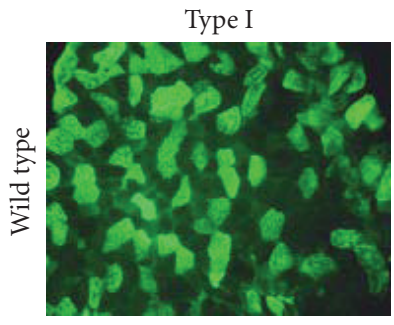

Type I
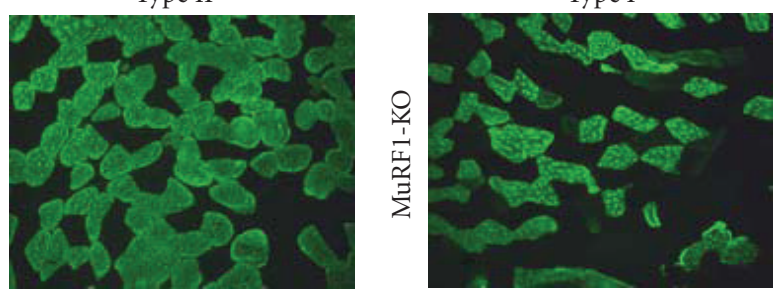

Type II

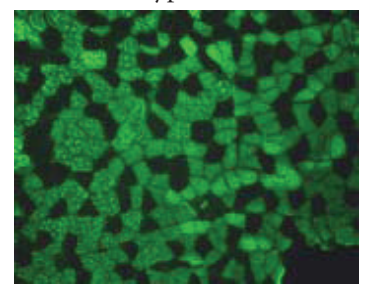

Type II

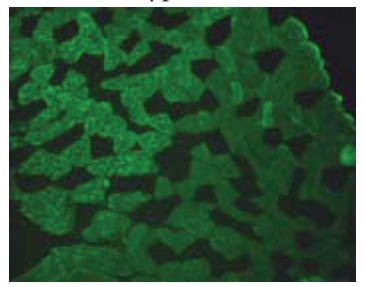

(b)

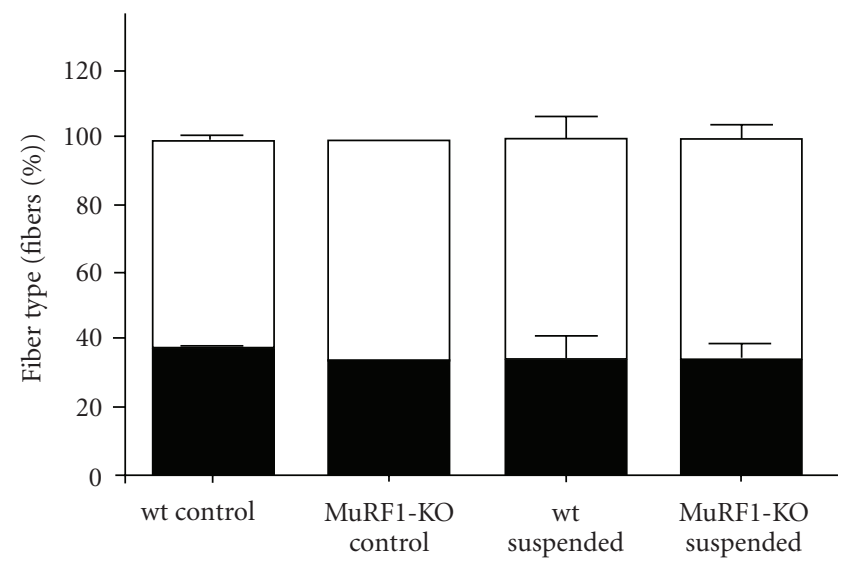

Type I

Type II

(d)

FIGURE 3: Fiber cross-sectional and fiber type analysis. (a) and (b): examples of cross-sections of soleus muscle stained for type I and type II fibers of wt (top row) and MuRF1-KO (bottom row) of control (left two columns) and suspended (right two columns) mice. (c): crosssectional area analysis; (d): fiber type analysis.

3.3. Altered Twitch Potentiation and Fatigability in MuRF1Deficient Soleus Muscle. We also measured the well-known phenomenon of twitch potentiation, by measuring twitch force prior to a tetanic stimulation and immediately after tetanic stimulation. Figure 5(a) shows an example of the used protocol. The increase in twitch force after the tetanus (i.e., twitch potentiation) has been shown to be due to increased myosin light chain 2 (MLC2) phosphorylation during the tetanus [20] which in turn increases calcium sensitivity. Interestingly hindlimb suspension results in a significant reduction in the twitch potentation of MuRF1$\mathrm{KO}$ mice (Figure 5(b)). This finding suggests that MuRF1 is likely to play a role in MLC2 phosphorylation, by either having an effect on the activity of the myosin light kinase or on the ability of the MLC2 to become phosphorylated. Additional experiments with phospho-specific antibodies will be required to further investigate this hypothesis.

Next, we studied the development of fatigue of soleus muscle by stimulation at a submaximal frequency $(60 \mathrm{~Hz}$ for 1 second) every three seconds for a total of 5 minutes. The mean data of all muscles are shown in Figure 6(a). The percent of maximum peak force for the first five stimuli and subsequent every fifth were plotted and fitted to a double sigmoidal curve. We found that the muscles from the hindlimb suspended mice had a significant difference between wt and MuRF1-KO mice in that the wt mice had significantly less fatigue than the $\mathrm{KO}$ mice. Figure 6(b) shows the force after 2 minutes of the fatigue protocol; the results clearly reveal that muscles from the KO suspended mice had fatigued significantly more than those from the wt suspended mice. 


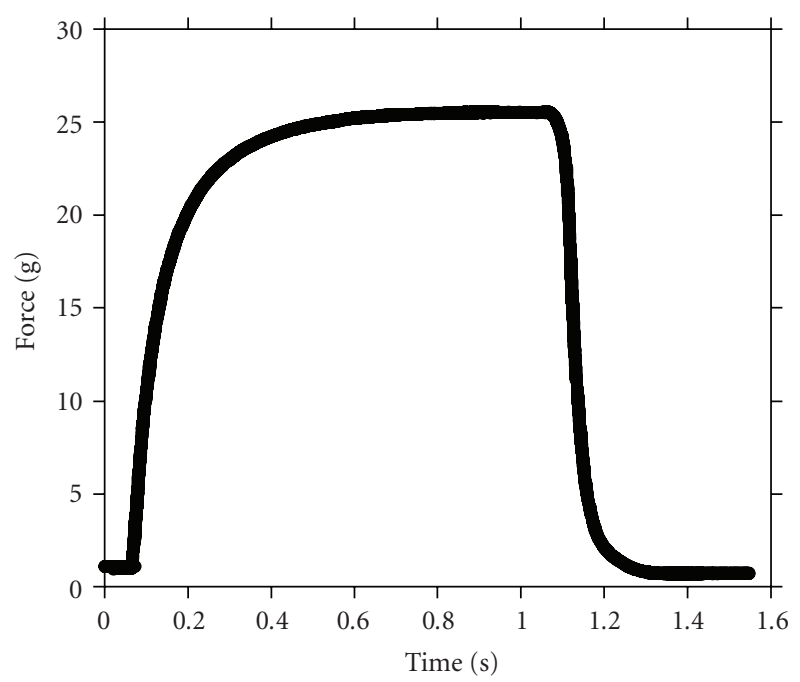

(a)

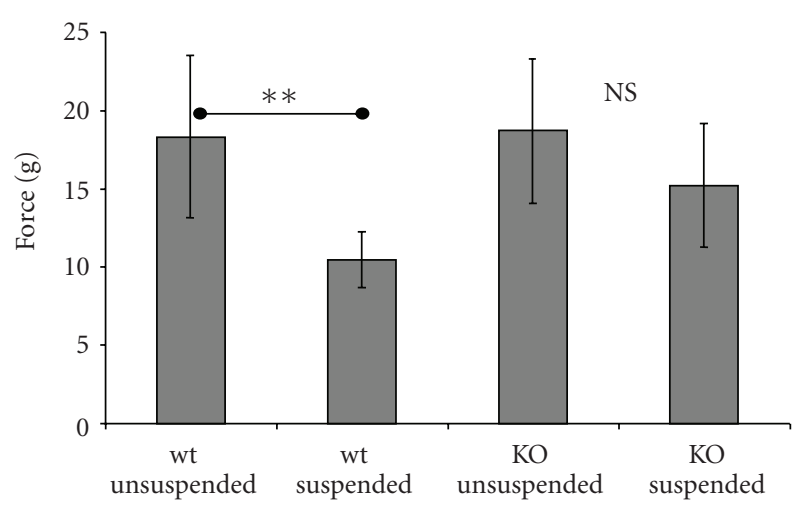

(b)

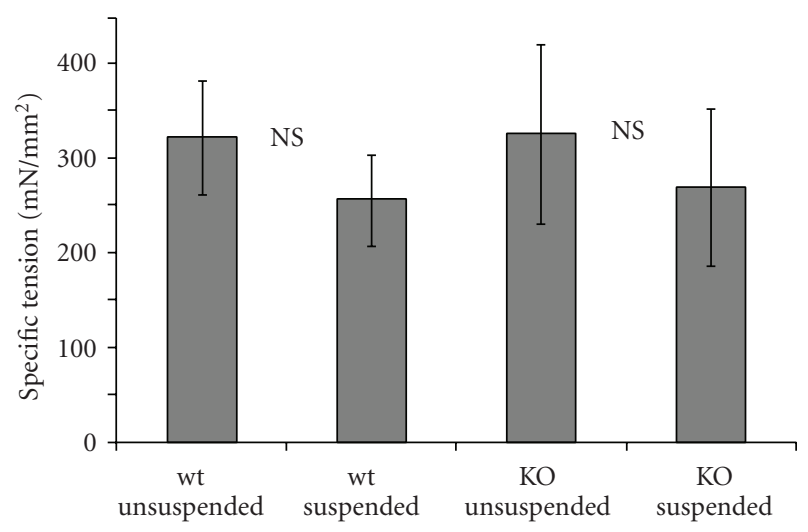

(c)

Figure 4: Tetanus Contraction. We stimulated the soleus muscle to a maximum tetanic contraction at a stimulus frequency of $150 \mathrm{~Hz}$. (a) Example of a tetanus of a wt mouse. (b) Mean results of wt and MuRF1-KO mice who had been suspended or unsuspended. There is a significant difference when comparing the absolute peak tetanic force. The data from wt unsuspended mice are significantly different from those of wt suspended mice. However, there is no significant force reduction between the KO unsuspended and the KO suspended mice. (c) Specific tetanic force comparisons did not reveal a significant difference.

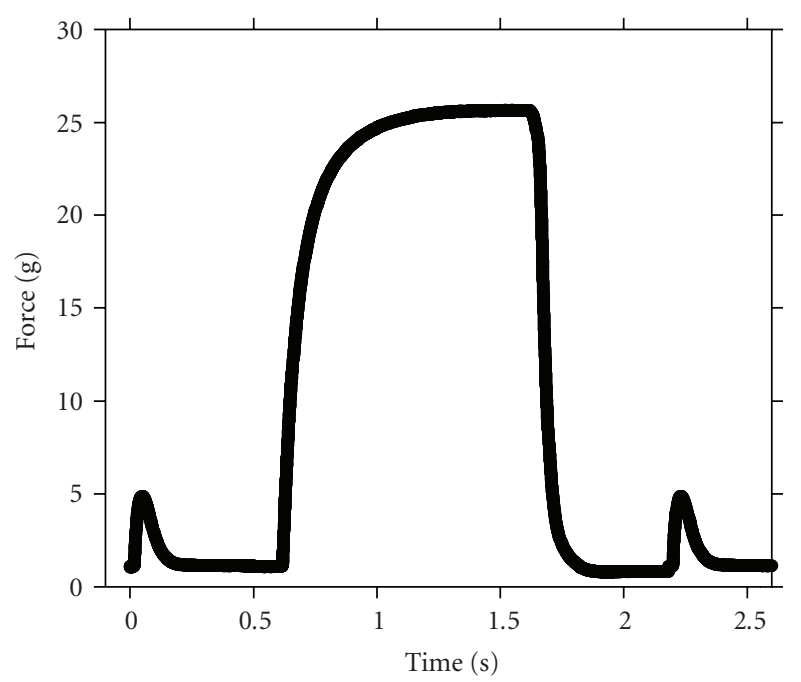

(a)

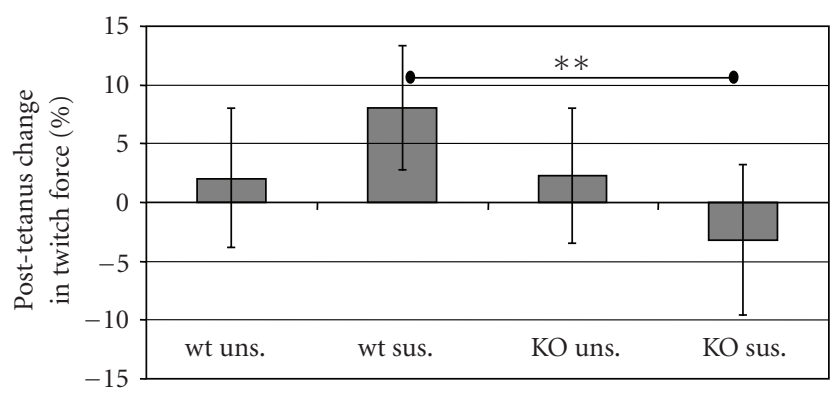

(b)

Figure 5: Twitch potentiation. (a) example of protocol. A twitch was elicited before and following a tetanus contraction and peak twitch force after contraction was compared to that before. (b) There is a significant difference between the wt suspended and KO suspended in twitch potentiation, indicating a difference in MLC phosophorylation between the wt and KO mice.

\section{Discussion}

4.1. MuRF1's Role in Atrophy. Previous studies on MuRF1's role in the development of muscle atrophy were carried out in a denervation (DEN) model in which the sciatic nerve was cut and the lower limb muscles were denervated in wt and MuRF1-KO mice [11]. Results showed that MuRF1-KO mice had a $36 \%$ reduction in atrophy after 14 days of denervation. Because denervation might activate catabolic pathways via inflammatory and stress-related pathways that in turn might overlap with MuRF1 signaling pathways (e.g., for shared TNF-alpha and MuRF1 signaling, see [21]) we used a hindlimb suspension model which induces atrophy but keeps innervation intact. Furthermore we limited our analysis to 10 days after suspension when atrophy is significant but not yet severe, and reaches $\sim 25 \%$ atrophy in wt mice in the here analyzed two muscle types, tibialis cranialis and soleus. We intentionally focused our analysis on this shortened time period in an attempt to potentially gain insights into physiological rather than purely pathophysiological roles of MuRF1. Furthermore, we focused in our analysis on soleus 


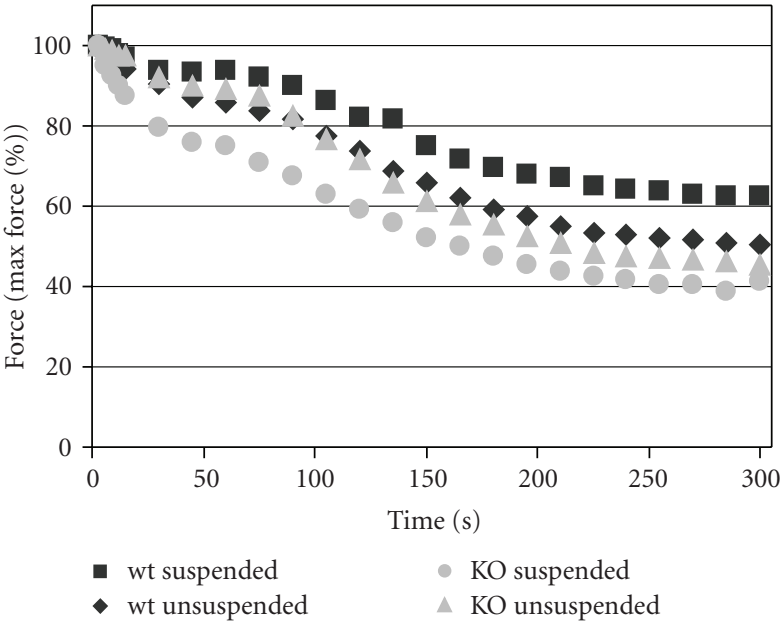

(a)

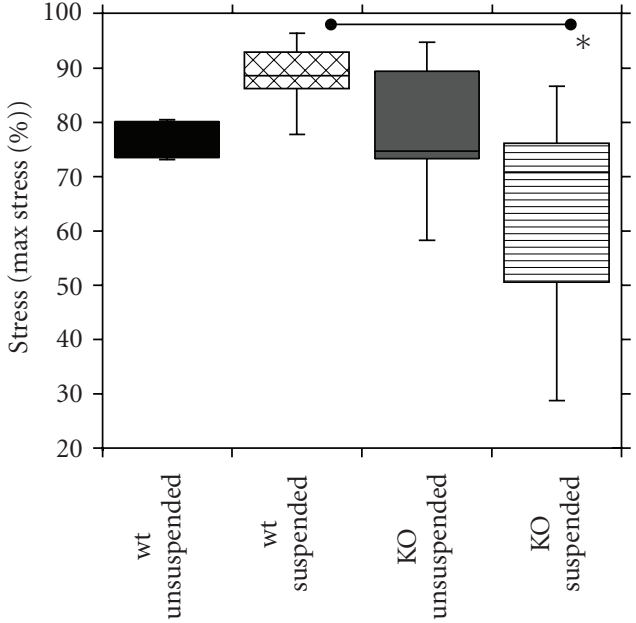

(b)

Figure 6: Muscle Fatigue. We fatigued the soleus muscle by stimulating the soleus muscle at a submaximal frequency (60 Hz, inset of Figure 5(a)) every three seconds for a total of 100 stimuli. (a) The mean force of all muscles. (b) Force at the 105 second time point. There is a significant difference between the wt suspended and the MuRF1-KO suspended mice.

muscle because it is one of the few muscles in the mouse with a high number of slow twitch fibers, making it possible to test for fiber-type differences in MuRF1-based muscle protection.

Future studies are required to address the effect of longer hindlimb suspension periods, extending for 2 weeks or longer. However, here, using this study design, we found a remarkable protection of the soleus muscle in the MuRF1$\mathrm{KO}$ mice during the tested 10-day-suspension period, displaying virtually complete protection (Table 1 and Figure 2). This high degree of protection is remarkable because MuRF1-KO muscle tissues will express other atrogenes such as atrogin-1, thought to be capable of catalyzing multiubiquitination and UPS-dependent degradation of muscle proteins $[6,12]$. Also interestingly, we could not detect a fiber-type dependence of MuRF1-based muscle protection here: both nonsuspended and suspended MuRF1-KO soleus tissues had comparable fiber type distributions, and both fiber type subpopulations were protected from atrophy in the absence of MuRF1 as shown by immunohistochemical typing as well as SDS-PAGE based MHC I/ MHC II quantification. This contrasts recent data that were obtained with the MuRF1-KO mice when using the denervation model: here, MuRF1 protein was preferentially induced in type-II fibers from the fast TA muscle. Consistent with an MuRF1 induction in type-II fibers by DEN, we found a preferential protection of type II fast fibers in the MuRF1-KO mice during DEN-induced atrophy [19]. Taken together, our data confirm an important role for MuRF1 during muscle atrophy development, but also stress the importance of using different atrophy induction models, and to analyze the effects that occur in fast and slow fiber type populations separately.

4.2. MuRF1 and Fatigue Control. Because we recently noted that MuRF1 overexpression causes metabolism effects we also compared wt and MuRF1-KO M soleus muscle with regards to their fatigue resistance when stressed by repetitive stimulations. Consistent with the idea that the role of MuRF1 extends to energy metabolism control [22], we found that inactivation of MuRF1 increases sensitivity to fatigue. A degradation or modification of contractile or regulatory proteins within the myofibril by MuRF1 is an unlikely explanation, because specific tetanic force and the force-frequency relation of suspended soleus muscle from wt and from MuRF1-KO mice were comparable (we also obtained similar findings from MuRF1-overexpressing MuRF1-TG mice, data not shown). Therefore, possibly MuRF1 signalling affects the control of oxidative phosphorylation, either indirectly by affecting mitochondrial functions (for an effect of MuRF1 overexpression on the mitochondrial matrix protein PDC, see $[14])$, or indirectly by affecting the ATP regenerating MCK enzyme activity [23]. Such a model where MuRF1 exerts its effects on muscle indirectly via its control of energy metabolism would also explain why overexpression of MuRF1 alone is not sufficient to cause a muscle atrophy but instead affectes energy metabolism [14], for a review see [24].

In our study we found significant differences in twitch potentiation between wt and MuRF1-KO muscle (Figure 5). Twitch potentiation can be explained as follows. The calcium that is released into the sarcoplasm when a muscle is tetanized not only activates the thin filament, allowing contraction to take place, but also activates the skeletal muscle $\mathrm{Ca}^{2+} /$ calmodulin-dependent myosin light chain kinase (skMLCK) to initiate phosphorylation of MLC2 (also known as regulatory light chain, RLC). MLC2 phosphorylation does not increase maximal tetanic force but increases force at submaximal calcium levels, such as occur during a twitch contraction. Thus after the tetanus has been terminated, the phosphorylation status of MLC2 is elevated for some time (ultimately phosphatases reduce the phosphorylation state back to baseline) and twitch force is potentiated [20]. We 
previously noted that MuRF1 interacts in the $\mathrm{Y} 2 \mathrm{H}$ system with MLC2 [16]. Possibly MuRF1 functions as an adapter protein to facilitate MLCK and MLC2 interactions. Because MuRF1 has been suggested to regulate the turnover of MLC-2 [13] it is also possible that the absence of MuRF1 negatively impacts the degree to which MLC2 is phosphorylated during tetanic stimulation. Alternatively MuRF1 might have an effect on skMLCK or on the phosphatase that dephosphorylates MLC2P. Future work is needed to establish the mechanisms by which MuRF1 deficiency reduced twitch potentiation in muscle from hindlimb suspended mice.

Taken together, our data indicate that the functions of MuRF1 extend beyond regulating myofibrillar protein breakdown, and include intricate fine-tunings of the phosphorylation status of MLC2 and metabolic properties of the skeletal muscle apparatus.

\section{Author's Contributions}

The first and the second authors contributed equally to this work.

\section{Acknowledgments}

The authors would like to thank Nick King for help with the hindlimb suspension experiments, Anselmo Moriscot for supervision of their fiber type studies, and Nicole Macha for excellent mouse breeding support. Supported by the DFG (La668/13-1), WI3278/2-1 (to C.C.W.) and the NAR University of Heidelberg (to S.L.), and by the NIH (HL062881 to H.G).

\section{References}

[1] M. J. Potthoff, E. N. Olson, and R. Bassel-Duby, "Skeletal muscle remodeling," Current Opinion in Rheumatology, vol. 19, no. 6, pp. 542-549, 2007.

[2] R. S. Lee-Young, B. J. Canny, D. E. Myers, and G. K. McConell, "AMPK activation is fiber type specific in human skeletal muscle: Effects of exercise and short-term exercise training," Journal of Applied Physiology, vol. 107, no. 1, pp. 283-289, 2009.

[3] D. A. Hood, "Mechanisms of exercise-induced mitochondrial biogenesis in skeletal muscle," Applied Physiology, Nutrition, and Metabolism, vol. 34, no. 3, pp. 465-472, 2009.

[4] C. T. Putman, X. Xu, E. Gillies, I. M. MacLean, and G. J. Bell, "Effects of strength, endurance and combined training on myosin heavy chain content and fibre-type distribution in humans," European Journal of Applied Physiology, vol. 92, no. 4-5, pp. 376-384, 2004.

[5] S. H. Lecker, R. T. Jagoe, A. Gilbert et al., "Multiple types of skeletal muscle atrophy involve a common program of changes in gene expression," FASEB Journal, vol. 18, no. 1, pp. 39-51, 2004.

[6] D. J. Glass, "Signalling pathways that mediate skeletal muscle hypertrophy and atrophy," Nature Cell Biology, vol. 5, no. 2, pp. 87-90, 2003.

[7] N. Frey, D. Frank, S. Lippl et al., "Calsarcin-2 deficiency increases exercise capacity in mice through calcineurin/NFAT activation," Journal of Clinical Investigation, vol. 118, no. 11, pp. 3598-3608, 2008.

[8] T. Snijders, L. B. Verdijk, and L. J. C. van Loon, "The impact of sarcopenia and exercise training on skeletal muscle satellite cells," Ageing Research Reviews, vol. 8, no. 4, pp. 328-338, 2009.

[9] S. H. Lecker, V. Solomon, W. E. Mitch, and A. L. Goldberg, "Muscle protein breakdown and the critical role of the ubiquitin-proteasome pathway in normal and disease states," Journal of Nutrition, vol. 129, no. 1, pp. 2275-2375, 1999.

[10] S. C. Bodine, E. Latres, S. Baumhueter, et al., "Identification of ubiquitin ligases required for skeletal muscle atrophy," Science, vol. 294, no. 5547, pp. 1704-1708, 2001.

[11] S. C. Bodine, E. Latres, S. Baumhueter, et al., "Identification of ubiquitin ligases required for skeletal Muscle Atrophy," Science, vol. 294, no. 5547, pp. 1704-1708, 2001.

[12] G. Mearini, S. Schlossarek, M. S. Willis, and L. Carrier, "The ubiquitin-proteasome system in cardiac dysfunction," Biochimica et Biophysica Acta, vol. 1782, no. 12, pp. 749-763, 2008.

[13] S. Cohen, J. J. Brault, S. P. Gygi et al., "During muscle atrophy, thick, but not thin, filament components are degraded by MuRF1-dependent ubiquitylation," Journal of Cell Biology, vol. 185, no. 6, pp. 1083-1095, 2009.

[14] S. Hirner, C. Krohne, A. Schuster et al., "MuRF1-dependent regulation of systemic carbohydrate metabolism as revealed from transgenic mouse studies," Journal of Molecular Biology, vol. 379, no. 4, pp. 666-677, 2008.

[15] C. C. Witt, S. H. Witt, S. Lerche, D. Labeit, W. Back, and S. Labeit, "Cooperative control of striated muscle mass and metabolism by MuRF1 and MuRF2," EMBO Journal, vol. 27, no. 2, pp. 350-360, 2008.

[16] S. H. Witt, H. Granzier, C. C. Witt, and S. Labeit, "MURF-1 and MURF-2 target a specific subset of myofibrillar proteins redundantly: towards understanding MURF-dependent muscle ubiquitination," Journal of Molecular Biology, vol. 350, no. 4, pp. 713-722, 2005.

[17] T. Centner, J. Yano, E. Kimura et al., "Identification of muscle specific ring finger proteins as potential regulators of the titin kinase domain," Journal of Molecular Biology, vol. 306, no. 4, pp. 717-726, 2001.

[18] C. C. Witt, S. H. Witt, S. Lerche, D. Labeit, W. Back, and S. Labeit, "Cooperative control of striated muscle mass and metabolism by MuRF1 and MuRF2," EMBO Journal, vol. 27, no. 2, pp. 350-360, 2008.

[19] A. S. Moriscot, I. L. Baptista, J. Bogomolovas et al., "MuRF1 is a muscle fiber-type II associated factor and together with MuRF2 regulates type-II fiber trophicity and maintenance," Journal of Structural Biology, vol. 170, no. 2, pp. 344-353, 2010.

[20] G. Zhi, J. W. Ryder, J. Huang et al., "Myosin light chain kinase and myosin phosphorylation effect frequency-dependent potentiation of skeletal muscle contraction," Proceedings of the National Academy of Sciences of the United States of America, vol. 102, no. 48, pp. 17519-17524, 2005.

[21] V. Adams, N. Mangner, A. Gasch et al., "Induction of MuRF1 is essential for TNF-alpha-induced loss of muscle function in mice," Journal of Molecular Biology, vol. 384, no. 1, pp. 48-59, 2008.

[22] S. Koyama, S. Hata, C. C. Witt et al., "Muscle RINGfinger protein-1 (MuRF1) as a connector of muscle energy metabolism and protein synthesis," Journal of Molecular Biology, vol. 376, no. 5, pp. 1224-1236, 2008. 
[23] M. S. Willis, M. Rojas, L. Li et al., "Muscle ring finger 1 mediates cardiac atrophy in vivo," American Journal of Physiology, vol. 296, no. 4, pp. H997-H1006, 2009.

[24] O. Mayans and S. Labeit, "MuRFs: specialized members of the TRIM/RBCC family with roles in the regulation of the trophic state of muscle and its metabolism," in TRIM/RBCC Proteins, G. Meroni, Ed., Landes Biosciences, 2010. 

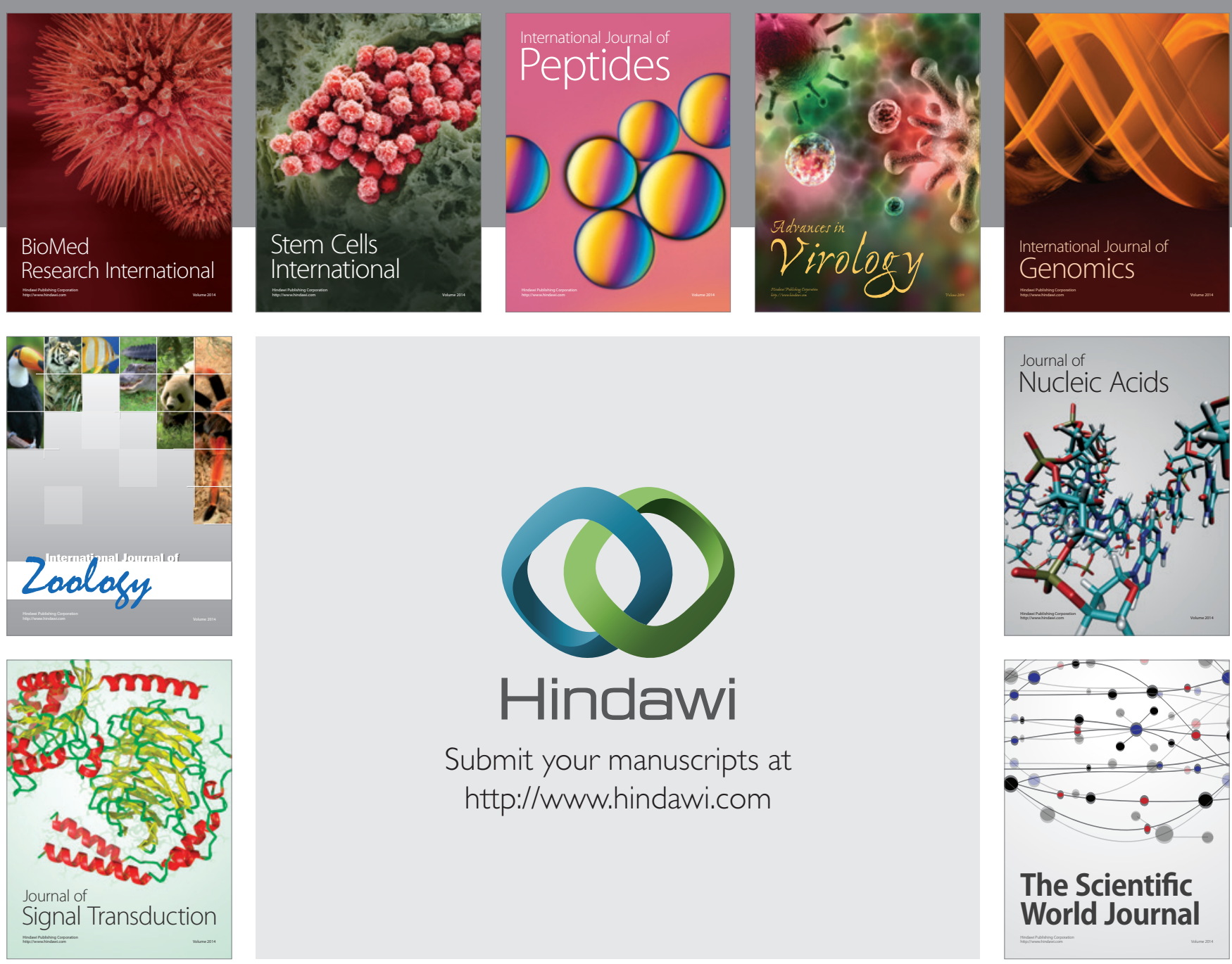

Submit your manuscripts at

http://www.hindawi.com
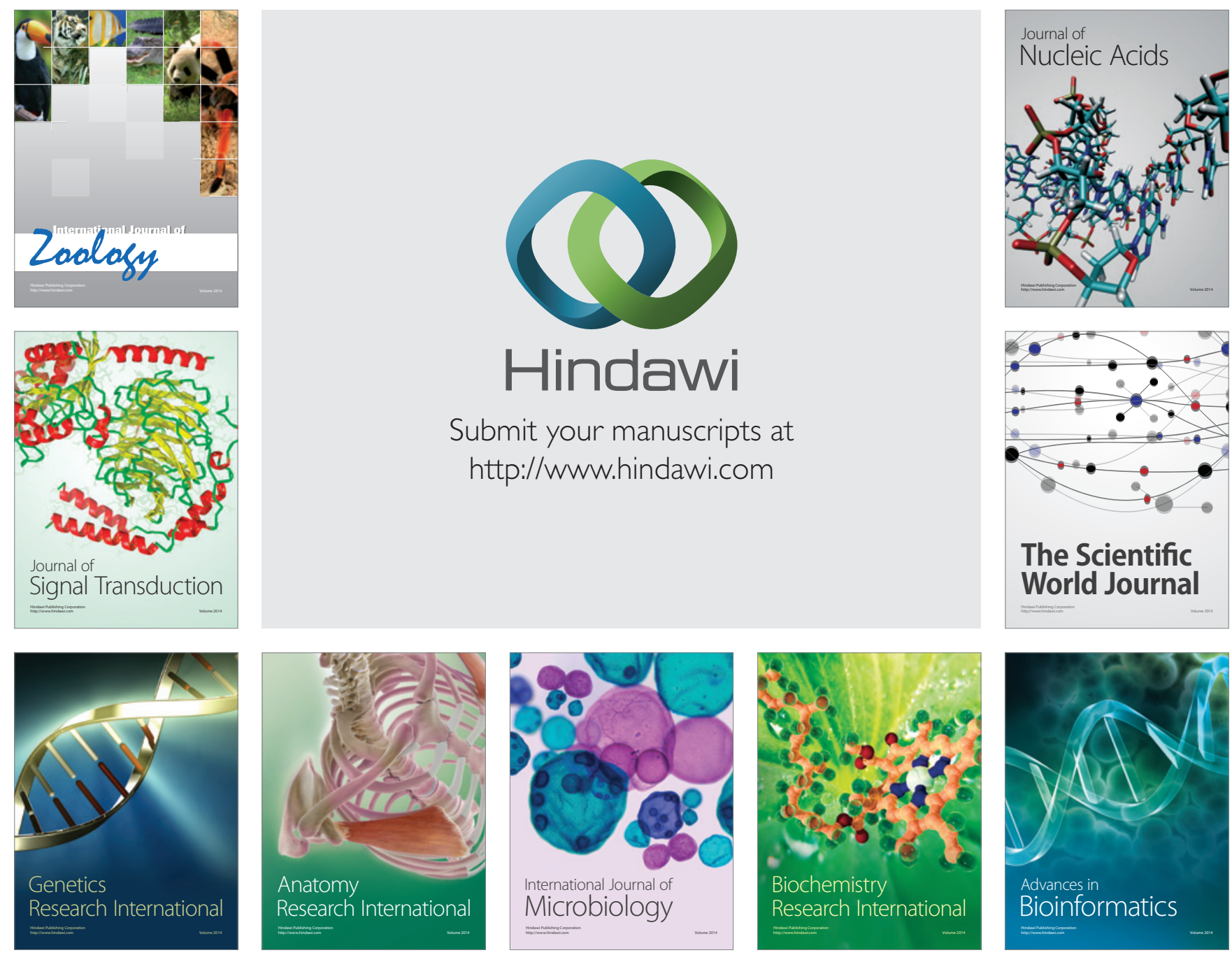

The Scientific World Journal
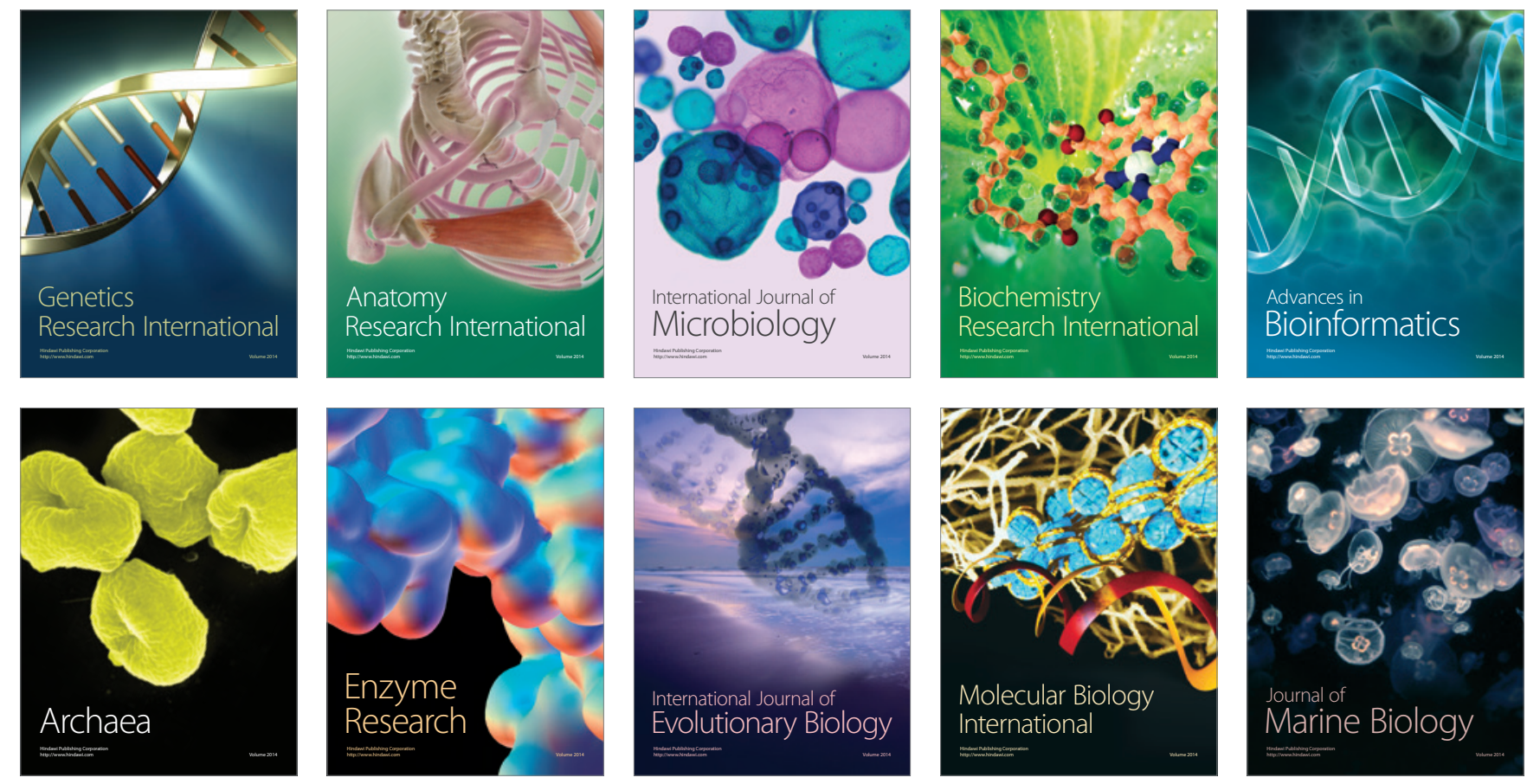